\title{
Chromosomal telomere attrition as a mechanism for the increased risk of epithelial cancers and senescent phenotypes in type 2 diabetes
}

\author{
M. J. Sampson • D. A. Hughes
}

Received: 27 January 2006 / Accepted: 24 April 2006 / Published online: 21 June 2006

(C) Springer-Verlag 2006

\begin{abstract}
Telomeres are the repeat DNA sequences at the end of chromosomes necessary for successful DNA replication and chromosomal integrity. Telomeres shorten at cell division at a rate determined by oxidative DNA damage, and cells are triggered into replicative senescence once telomeres shorten to a critical length. Telomere-related chromosomal maintenance also has a role in carcinogenesis. Type 2 diabetes is characterised by increased oxidative stress, increased oxidative DNA damage, senescent retinal and renal phenotypes, and an increased risk of epithelial malignancy. We suggest that increased oxidative DNA damage and telomere attrition in type 2 diabetes leads to: (1) carcinogenic telomere-dependent chromosomal nonreciprocal translocations, genomic instability, and the development of epithelial cancers; (2) senescent retinal and renal phenotypes (expressed as diabetic retinopathy and nephropathy); and (3) senescent vascular endothelial, monocyte-macrophage and vascular smooth muscle cells (expressed as endothelial dysfunction and accelerated atherogenesis). An adverse intrauterine environment leads to increased feto-placental oxidative stress and feto-placental oxidative DNA damage. We also suggest that intrauterine oxidative DNA damage and telomere shortening is another point at which increased oxidative stress could contribute to a pre-programmed increased risk of senescent
\end{abstract}

M. J. Sampson $(\square)$

School of Medicine, Health Policy and Practice,

University of East Anglia,

Norwich NR4 7UA, UK

e-mail: mike.sampson@nnuh.nhs.uk

D. A. Hughes

Institute of Food Research, Norwich Research Park,

Norwich, UK phenotypes in adult offspring, characterised by type 2 diabetes and epithelial malignancy. These suggestions can be used to understand early glucose intolerance in the young children of type 1 diabetes pregnancies, poor cancer outcomes in type 2 diabetes, beta cell fatigue in type 2 diabetes and the absence of increased epithelial cancer risk in type 1 diabetes.

Keywords Atherosclerosis - Cancer DNA · Oxidative stress $\cdot$ Pre-programming $\cdot$ Senescence .

Telomere · Type 2 diabetes

\section{Abbreviations \\ IUGR intrauterine growth retardation \\ NRT non-reciprocal translocation \\ RPE retinal pigment epithelial cells}

\section{Introduction and background}

Eukaryotic chromosomes are capped with telomeres, tandem repeats of the DNA sequence TTAGGG extending over 6 to $15 \mathrm{~kb}$, which are necessary for successful DNA replication and chromosomal integrity [1]. Telomeres in somatic human cells shorten at cell division, and once shortened to a critical length cells are triggered into replicative senescence [2], an irreversible cell cycle block in G0/G1. Cells trapped in replicative senescence function differently, and some senescent cell types are more likely to undergo apoptosis if exposed to continued oxidative damage [3-5]. Rates of telomere shortening at cell division are highly dependent on oxidatively induced strand breaks in telomeric DNA, on cellular oxidant balance, and on the protection offered by the reverse transcriptase telomerase 
and other telomere-maintenance proteins [6]. The GGG sequence in telomeres is particularly prone to DNA damage, and telomeric DNA is more prone to oxidatively induced single-strand breaks than non-telomeric DNA [7]. Recently, we have shown both that type 2 diabetes is characterised by increased susceptibility to oxidative DNA damage and that telomere attrition is directly related to levels of oxidative DNA damage in monocytes $[8,9]$. Epithelial cells with substantial telomeric disruption are more prone to develop chromosomal disorganisation and carcinogenic chromosomal non-reciprocal translocations [NRTs] in epithelial cells $[10,11]$. Recent surprising observations have confirmed an increased risk of many cancers in type 2 diabetes [12], in addition to the established renal, retinal and vascular complications, and the mechanisms underlying this association remain unclear. A substantial and separate literature also supports an association between an adverse intrauterine environment and the later development in offspring of an increased risk of atherogenesis, hypertension and type 2 diabetes [13-15]. Feto-placental telomere attrition has attracted interest as a hypothetical bridge between the intrauterine environment and these conditions, although much of this interest is derived from in vitro models rather than clinical or epidemiological studies [3-5].

\section{For debate}

We suggest that increased oxidative DNA damage in type 2 diabetes leads to telomeric DNA damage in many cell types, to accelerated telomere shortening at cell division, and to senescent phenotypes in multiple cell types. We suggest that this leads to: (1) the development of chromosomal NRTs, genomic instability and an increased risk of epithelial malignancies; (2) accelerated endothelial and vascular cell senescence (expressed as endothelial dysfunction and atherogenesis); (3) progressive beta cell senescence (expressed as beta cell failure); and (4) senescent renal and retinal phenotypes (expressed as diabetic nephropathy and retinopathy). Separately, as an adverse intrauterine environment leads to increased feto-placental oxidative stress and DNA damage, we suggest that this leads to telomere attrition in multiple fetal cell types, preprogramming offspring towards a senescent adult phenotype of endothelial dysfunction, atherogenesis, pancreatic beta cell senescence and type 2 diabetes. Pre-programmed telomere shortening in epithelial cells would predispose this phenotype towards an increased risk of epithelial-derived malignancies. These testable suggestions could also account for some of the less understood senescent characteristics associated with type 2 diabetes and are compatible with much existing data.

\section{Adverse intrauterine environment, oxidative DNA damage and telomere attrition}

Increased oxidative stress and oxidative damage to fetoplacental DNA and fetal vascular endothelium mediate drug-, ethanol- and diabetes-induced embryopathies in animal models [16]. An adverse intrauterine environment and intrauterine growth retardation [IUGR] are also associated with increased maternal, fetal and placental oxidative DNA damage [17]. Telomere length in somatic cells originates from telomere-length programming in the germ line [18] and telomere maintenance by the reverse transcriptase telomerase is downregulated in the later stages of fetal development, in some fetal tissues, and in placental tissues in IUGR [19, 20]. Increased feto-placental oxidative DNA damage in an adverse intrauterine environment could pre-programme multiple fetal cell types towards telomere attrition and replicative senescence, as it is biologically highly plausible that increased oxidative stress and DNA damage in the feto-placental unit translates into fetal telomere damage and shortening $[1,2,7]$. If this were the case, one would expect the newborn and infant offspring born from an adverse intrauterine environment to demonstrate (a) senescent phenotype(s). Many groups have in fact made the striking observation that the very young children of mothers with pre-conception type 1 diabetes (an archetype for a severely adverse intrauterine environment) have a prematurely aged phenotype characterised by glucose intolerance, endothelial dysfunction, early markers of atherogenic risk and psychomotor deficits [21].

\section{Telomere attrition and increased malignancy risk in type 2 diabetes}

The consistently increased risk of many epithelial cancers, particularly gastro-intestinal cancers, in type 2 diabetes has been reviewed recently [12]. Although confounding by obesity and dietary factors is possible, this association remains unexplained. Telomeric integrity is necessary for genomic stability, and telomere-length abnormalities in epithelial cells are an extremely early observation in the development of many epithelial-derived tumours [22], although there is a lack of prospective epidemiological evidence linking antecedent telomere attrition with increased cancer risk [23]. However, the association between epithelial telomere-length abnormalities and the earliest stages of epithelial cancer development, including the transition from adenoma to carcinoma in colorectal epithelial cells, has often been observed [22, 24]. Progressive telomere attrition and replicative senescence in epithelial cells could be seen as a tumour-suppressor mechanism, as cells triggered into senescence are unresponsive to growth 
factors, and have no mitotic potential [10, 11, 25]. However, there is now significant evidence that telomere shortening during cell proliferation promotes genomic changes that predispose towards the development of epithelial cancers. In particular, telomere shortening in animal and human models promotes epithelial cell tumours through the chromosomal fusion-bridge breaking NRTs of chromosomal material and genomic instability which characterise carcinogenesis [10, 11, 25-27], and particularly epithelial carcinogenesis [10]. Data have appeared supporting a linkage between the intrauterine environment, increased fetal DNA damage and later cancer risk in animal models [28] and in humans [29, 30], although the data in humans are conflicting and confounded by obesity [30]. We suggest that the development of type 2 diabetes with increasing oxidative stress leads to oxidative DNA damage $[9,10]$ in mitotically active epithelial cells, leading to telomere attrition and an increased risk of epithelial cell tumours through fusion-bridge breaking, NRTs and genomic instability [10,11, 25-27]. The poorer outcomes in type 2 diabetes patients for recurrence-free survival rates and median survival [12] could also be related to this hypothesis, with type 2 diabetes driving a progressive repopulation of carcinogenic genomically unstable epithelial cells $[10,11,25-27]$. In this context, it is important to note that evidence of a substantially increased risk of epithelial cancers in type 1 diabetes is lacking [12], and that we and others have shown that increased DNA oxidative susceptibility is not a feature of type 1 diabetes [31], perhaps because of more effective DNA repair mechanisms in younger type 1 patients [32]. We also suggest, although the epidemiological evidence is lacking, that an adverse intrauterine environment could be one variable that predisposes towards both type 2 diabetes and an increased risk of epithelial malignancies through feto-placental pre-programming of epithelial and other cell types mediated through telomere attrition.

It should be stressed that there are other proposed mechanisms for the association between some epithelial cancers and type 2 diabetes. Firstly, one potential confounding variable is exposure to an antecedent diet or lifestyle that contributes to an increased risk of both type 2 diabetes and cancer [33,34]. Secondly, obesity may be an independent contributor to both conditions [34]. Another dominant hypothesis, for colorectal malignancy in particular, is that hyperinsulinaemia and increased insulin-like growth factors in type 2 diabetes or insulin-resistant populations have a direct action on colonic mucosal cell proliferation, differentiation and carcinogenesis. This has been well reviewed recently, although causality is not established [33, 34]. These mechanisms and the present suggestions are not mutually exclusive, and there may in fact be some overlap. Recently, it has been shown that increasing insulin resistance associated with weight gain [35], or peripheral insulin resistance [36], are significantly inversely associated with leucocyte telomere length, and that increased oxidative stress and telomere damage may be one contributor to these observations $[35,36]$.

\section{Telomeres, replicative senescence and atherogenesis in type 2 diabetes}

Endothelial dysfunction may precede the development of type 2 diabetes, peripheral insulin resistance and atherogenesis, and while endothelial dysfunction may antedate and contribute to these diseases it is unclear why it should develop initially [37, 38]. The phenotype of dysfunctioning vascular endothelial cells in these conditions is very similar to the senescent endothelial phenotype described in vitro [39], and the role of telomere attrition as a pre-programming mechanism towards endothelial senescence and dysfunction has been reviewed [4-6]. We suggest that increased oxidative DNA and telomere damage in type 2 diabetes, promotes a characteristic endothelial senescence and dysfunction, monocyte-macrophage and vascular smooth cell senescence and dysfunction, and promotes atherogenesis. We also suggest that an adverse intrauterine environment and feto-placental DNA damage and telomere attrition could be a contributor towards an adult phenotype of endothelial dysfunction associated with type 2 diabetes and beta cell failure in some subjects.

\section{Telomere attrition and beta cell replicative senescence}

Progressive pancreatic beta cell senescence and failure are early features of type 2 diabetes, and hyperglycaemia leads to progressive loss of beta cell mass with increased rates of beta cell senescence and apoptosis, in part mediated through oxidative stress [40]. Beta cell telomere shortening predicts the risk of beta cell growth arrest and senescence in human adult islet cell cultures, where telomere erosion appears to occur at a faster rate than in other cell lines [41]. In animal models, IUGR predisposes to fetal mitochondrial dysfunction, increased reactive oxygen species (ROS) generation, mitochondrial DNA damage, and progressive beta cell failure in the adult offspring [42]. Increased mitochondrial ROS generation should also translate to beta cell chromosomal DNA and telomere damage in IUGR models. Oxidative DNA damage and upregulated DNA repair mechanisms are recognised in the beta cell in type 2 diabetes [43], and there is an inverse relationship between beta cell volume density and levels of DNA oxidation products in human type 2 diabetes [44]. We suggest that type 2 diabetes leads to accelerated oxidative damage to 
beta cell telomeric DNA, with progressive beta cell senescence and failure. This suggestion is compatible with the natural history of beta cell failure in type 2 diabetes, and with the unexplained failure of beta cell mass to increase in response to progressive hyperglycaemia [40]. We also suggest that one contributor to later beta cell senescence and failure in some subjects could be antecedent fetal preprogramming due to increased feto-placental oxidative stress and telomeric DNA damage in IUGR pregnancies.

\section{Telomeres, replicative senescence and diabetic nephropathy}

An adverse intrauterine environment leads to reduced renal glomerular numbers in offspring of animal models and humans, and to an increased risk of glomerular hyperfiltration, proteinuria and hypertension [45]. Accelerated renal cortical cell senescence and telomere shortening have also been described in humans and in animal models of renal disease in relation to an adverse fetal environment by Hales and colleagues [46]. The phenotype of diabetic nephropathy in type 2 diabetes is characterised by a high prevalence of non-progressive low-level proteinuria ('microalbuminuria') close to diagnosis of type 2 diabetes and associated for unknown reasons with endothelial dysfunction, exceptionally high rates of associated cardiovascular disease and little evidence of a significant genetic contribution [47]. We suggest that increased renal oxidative DNA damage in type 2 diabetes, a predictor of diabetic nephropathy [48], is associated with telomere damage and attrition, which allows early expression of a renal phenotype of progressive glomerular cell senescence and proteinuria associated with accelerated endothelial and vascular cell senescence and atherogenesis. We also suggest that subjects with an antecedent history of IUGR, programmed towards reduced glomerular numbers [45, 46], would be particularly prone to developing a senescent renal cortical phenotype if they developed type 2 diabetes. The observation of more effective DNA repair mechanisms in type 1 diabetes, with little evidence of increased oxidative DNA susceptibility compared with type 2 diabetes [31,32], could account for the earlier onset of a senescent renal phenotype and proteinuria in type 2 diabetes than in type 1 diabetes.

\section{Telomeres, replicative senescence and diabetic retinopathy}

Endothelial dysfunction (and presumably retinal vascular endothelial dysfunction) is closely and directly related to the risk of diabetic retinopathy [49]. Retinal pigment epithelial (RPE) cells have a central role in maintaining retinal matrix and macular structure and function [50-52]. Damaged RPE cells demonstrate increased telomere shortening and senescence $[53,54]$. We suggest that development of type 2 diabetes promotes oxidative DNA damage and telomere-associated senescence in retinal endothelial and RPE cells, and the senescent phenotype of diabetic retinopathy. This suggestion would fit with the particular risk of RPE-dependent maculopathy in type 2 diabetes, and the independent association between retinopathy and adverse cardiovascular outcomes [55]. The observation of more effective DNA repair mechanisms in type 1 diabetes, with little evidence of increased oxidative DNA susceptibility compared with type 2 diabetes [31, 32] could account for the earlier onset of a senescent retinal phenotype in type 2 diabetes compared with type 1 diabetes.

\section{Existing data in type 2 diabetes and insulin resistance}

We have shown an inverse association between oxidative DNA damage and telomere length in circulating mononuclear cells in type 2 diabetes $(r=-0.55 ; p=0.018)$, and increased oxidative DNA damage in type 2 diabetes [8, 9], both of which support the necessary central argument for the link between oxidative DNA damage and telomere attrition. Aviv et al. [36] recently described a significant inverse relationship $(r=-0.149 ; p=0.001)$ between leucocyte telomere length (adjusted for age and smoking) and measures of peripheral insulin resistance in 833 premenopausal women, although this relationship was absent in 683 postmenopausal women [36]. Similar inverse relationships between leucocyte telomere length and increasing peripheral insulin resistance $(r=-0.47 ; p<0.01)$ were described in 70 subjects in the Bogalusa Heart study cohort [35], and in South Asian populations ( $r=-0.4$; $p=0.01 ; n=40$ ) without type 2 diabetes [56]. The latter group also described lower leucocyte telomere length $(p=0.00001)$ in a South Asian type 2 diabetes group compared with controls [56]. Increased oxidative stress was suggested as one possible contributor to leucocyte telomere shortening in these groups [35, 36, 56], and this would be compatible with the present hypothesis.

\section{Implications}

These testable suggestions are to some degree independent of existing models for associations between cancer and type 2 diabetes, between oxidative stress and the complications of type 2 diabetes, and between the intrauterine environment and some adult phenotypes. The difficulties in testing these suggestions will be in distinguishing between pre-programming models of telomere shortening and downstream 
attritional models where diabetes or atherosclerosis influence telomere attrition. In particular, the potential confounding effects of obesity on these associations should be emphasised. To distinguish between these models we need (1) large, long-term, prospective epidemiological studies on telomere length and maintenance from infancy onwards in normal populations, linked to disease registries (cancer and diabetes) and to recorded birthweight; and (2) retrospective analyses of telomere length in stored telomere DNA in large disease registries (cancer, diabetes and diabetes complications) linked to birthweight data. In the short-term, we predict ten testable consequences of these suggestions:

(1) Telomere length in cord blood leucocyte and circulating vascular endothelial cells will be significantly lower at delivery in small-for-gestational-age offspring who have experienced an adverse intrauterine environment, compared with controls matched for gestational age.

(2) Telomere length in peripheral blood leucocyte, skin fibroblasts and epithelial cells in healthy young children and adolescents will be independently and inversely related to the later risk of developing both type 2 diabetes and epithelial cell malignancy, and directly to birthweight.

(3) Telomere length in bone marrow-derived circulating progenitor vascular endothelial cells, repopulating the vascular endothelium, will be independently and inversely related to biomarkers of endothelial dysfunction in adults.

(4) Telomere length in peripheral blood leucocyte, skin fibroblast and epithelial cells in existing elderly population cohorts with recorded birthweight but without type 2 diabetes or vascular disease will be directly and independently related to birthweight.

(5) Cross-sectional studies will demonstrate shorter telomere length in leucocytes, skin fibroblasts and epithelial cells from non-diabetic subjects in the upper quartile of the peripheral insulin-resistance range, independently of confounding by inflammatory markers.

(6) Low birthweight will be an independent predictor of increased risk of diabetic nephropathy and retinopathy, the early appearance of these conditions, and of early beta cell failure in type 2 diabetes.

(7) Telomere length in the colonic epithelial cells and skin fibroblasts of subjects with type 2 diabetes will be significantly shorter than those without type 2 diabetes, and age-related decline in telomere length will accelerate after the development of type 2 diabetes compared with controls.

(8) Telomere length in type 2 diabetes populations will be independently and inversely associated with the prospective risk of developing microvascular complications in type 2 diabetes, and with their rate of progression to sight-threatening diabetic eye disease and macroproteinuria.

(9) Incident cancer cases will increase sharply after incident diagnosis of diabetes cases in large cancer registries.

(10) Functional polymorphisms in telomere-maintenance genes will be independent contributors to: risk of type 2 diabetes; rates of beta cell failure in type 2 diabetes; the incidence and prevalence of diabetic nephropathy and diabetic retinopathy; and adverse vascular outcomes in type 2 diabetes.

Acknowledgements We thank Dr K. Dhatariya, Dr Dozio and Dr R. Temple (Norfolk and Norwich University Hospital) and Professor Dylan Edwards (School of Biological Sciences, University of East Anglia) for commenting on this manuscript. We thank the Norwich and Norfolk Diabetes Trust, and the Biotechnology and Biological Sciences Research Council, UK for financial support.

\section{References}

1. von Zglinicki $\mathrm{T}$ (2002) Oxidative stress shortens telomeres. Trends Biochem Sci 27:339-344

2. von Zglinicki T (2000) Role of oxidative stress in telomere length regulation and replicative senescence. Ann N Y Acad Sci 908:99-110

3. Stefanec T (2004) How the endothelium and its bone marrow derived progenitors influence development of disease. Med Hypotheses 62:247-251

4. Demerath EW, Cameron N, Gillman MW (2004) Telomeres and telomerase in the fetal origins of cardiovascular disease: a review. Hum Biol 76:127-134

5. Edo MD, Andres V (2005) Aging, telomeres and atherosclerosis. Cardiovasc Res 66:213-221

6. Savage SA, Stewart BJ, Eckert A, Kiley M, Liao JS, Chanock SJ (2005) Genetic variation, nucleotide diversity, and linkage disequilibrium in seven telomere stability genes suggest that these genes may be under constraint. Hum Mutation 26:343-350

7. Petersen S, Saretzki G, von Zglinicki T (1998) Preferential accumulation of single-stranded regions in telomeres of human fibroblasts. Exp Cell Res 239:152-160

8. Sampson MJ, Astley S, Richardson T et al (2001) Increased DNA oxidative susceptibility without increased plasma LDL oxidizability in type II diabetes: effects of alpha-tocopherol supplementation. Clin Sci (Lond) 101:235-241

9. Sampson MJ, Winterbone M, Hughes JC (2006). Monocyte telomere shortening and oxidative DNA damage in type 2 diabetes. Diabetes Care 29:283-289

10. Hanahan D (2000) Benefits of bad telomeres. Nature 406: 573-574

11. Artandi SE, Chang S, Lee SW (2000) Telomere dysfunction promotes reciprocal translocations and epithelial cancers in mice. Nature 406:641-644

12. Richardson LC, Pollack LA (2005) Therapy insight: influence of type 2 diabetes on the development, treatment and outcomes of cancer. Nat Clin Prac Oncol 2:48-53

13. Godfrey KM, Barker DJ(2000) Fetal nutrition and adult disease. Am J Clin Nutr 71:1344S-1352S

14. Sattar N (2004) Do pregnancy complications and CVD share common antecedents. Atheroscler Suppl 5:3-7 
15. Hattersley AT, Tooke JE (1999) The foetal insulin hypothesis: an alternative explanation of the association of low birth weight with diabetes and vascular disease. Lancet 353:1789-1792

16. Wiktor H, Kankofer M, Schmerold I et al (2004) Oxidative DNA damage in placentas from normal and pre-eclamptic pregnancies. Virchows Arch 445:74-78

17. Takagi Y, Nikaido T, Toki T et al (2004) Levels of oxidative stress and redox-related molecules in the placenta in pre-eclampsia and fetal growth restriction. Virchows Arch 444:49-55

18. Bekaert S, Derradji H, Baatout S (2004) Telomere biology in mammalian germ cells and during development. Dev Biol 274:15-30

19. Kudo T, Izutsu T, Sato T (2000) Telomerase activity and apoptosis as indicators of ageing in placenta with and without intrauterine growth retardation. Placenta 21:493-500

20. Ulaner GA, Giudice LC (1997) Developmental regulation of telomerase activity in human fetal tissues during gestation. Mol Hum Reprod 3:769-773

21. Plagemann A, Harder T, Kohlhoff R et al (1997) Glucose tolerance and insulin secretion in children of mothers with pregestational IDDM or gestational diabetes. Diabetologia 40:1094-1100

22. Meeker AK, Hicks JL, Iacobuzio-Donahue CA et al (2004) Telomere length abnormalities occur early in the initiation of epithelial carcinogenesis. Clin Cancer Res 10:3317-3326

23. Wu X, Amos CI, Zhu Y et al (2003) Telomere dysfunction: a potential cancer predisposition factor. J Natl Cancer Inst 95:1211-1218

24. Plentz RR, Wiemann SU, Flemming P et al (2003) Telomere shortening of epithelial cells characterises the adenoma-carcinoma transition of human colorectal cancer. Gut 52:1304-1307

25. Greenberg RA (2005) Telomeres, crisis and cancer. Curr Mol Med 5:213-218

26. Soler D, Genesca A, Arnedo G, Egozcue J, Tusell L (2005) Telomere dysfunction drives chromosomal instability in human mammary epithelial cells. Genes Chromosomes Cancer 44:339-350

27. Hahn WC (2005) Telomere and telomerase dynamics in human cells. Curr Mol Med 5:227-231

28. Chen CS, Wells PG (2004) In utero origins of cancer: maternal dietary vitamin E, fetal oxidative DNA damage, and postnatal carcinogenesis in p53 knockout mice. Ann N Y Acad Sci 1031:395-398

29. Sallout B, Walker B (2003) The fetal origin of adult diseases. J Obstet Gynaecol 23:555-560

30. Ozanne SE, Fernandez-Twinn D, Hales CN (2004) Fetal growth and adult disease. Semin Perinatol 28:81-87

31. Astley S, Langrish-Smith A, Southon S, Sampson MJ (1999) Vitamin E supplementation and oxidative damage to DNA and plasma LDL in type 1 diabetes. Diabetes Care 22:1626-1631

32. Varvarovska J, Racek J, Stetina R et al (2004) Aspects of oxidative stress in children with type 1 diabetes mellitus. Biomed Pharmacother 58:539-545

33. Komninou D, Ayonote A, Richie JP, Rigas B (2003) Insulin resistance and its contribution to colon carcinogenesis. Exp Biol Med 228:396-405

34. Giovannucci E (2001) Insulin, insulin-like growth factors and colon cancer: a review of the evidence. J Nutr 131:3109S-3120S

35. Gardner JP, Li S, Srinivasan SR et al (2005) Rise in insulin resistance is associated with escalated telomere attrition. Circulation 111:2171-2177

36. Aviv A, Valdes A, Gardner JP, Swaminathan R, Kimura M, Spector TD (2006) Menopause modifies the association between leukocyte telomere length with insulin resistance and inflammation. J Clin Endocrinol Metab 91:635-640
37. Pinkney JH, Stehouwer CD, Coppack S, Yudkin J (1997) Endothelial dysfunction: cause of the insulin resistance syndrome. Diabetes 46(Suppl 2):S9-S13

38. Schalkwijk CG, Stehouwer CD (2005) Vascular complications in diabetes mellitus: the role of endothelial dysfunction. Clin Sci (Lond) 109:143-159

39. Minamino T, Miyauchi H, Yoshida T, Tateno K, Kunieda T, Komuro I (2004) Vascular cell senescence and vascular aging. J Mol Cell Cardiol 36:175-183

40. Masiello P (2006) Animal models of type 2 diabetes with reduced pancreatic beta-cell mass. Int J Biochem Cell Biol 38:873-893

41. Halvorsen TL, Beattie GM, Lopez AD, Hayek A, Levine F (2000) Accelerated telomere shortening and senescence in human pancreatic cells stimulated to divide in vitro. J Endocrinol 166:103-109

42. Simmons RA, Suponitsky-Kroyter I, Selak MA (2005) Progressive accumulation of mitochondrial DNA mutations and decline in mitochondrial function lead to beta-cell failure. J Biol Chem 280:28785-28791

43. Tyrberg B, Anachkov KA, Dib SA, Wang-Rodriguez J, Yoon KH, Levine F (2002) Islet expression of the DNA repair enzyme 8oxoguanosine DNA glycosylase (Ogg1) in human type 2 diabetes. BMC Endocr Disord 2:2

44. Sakuraba H, Mizukami H, Yagihashi N, Wada R, Hanyu C, Yagihashi S (2002) Reduced beta-cell mass and expression of oxidative stress-related DNA damage in the islet of Japanese type II diabetic patients. Diabetologia 45:85-96

45. Mackenzie HS, Brenner BM (1995) Fewer nephrons at birth: a missing link in the etiology of essential hypertension? Am J Kidney Dis 26:91-98

46. Jennings BJ, Ozanne SE, Dorling MW, Hales CW (1999) Early growth determines longevity in male rats and may be related to telomere shortening in the kidney. FEBS Lett 448:4-8

47. Mogensen CE (2003) Microalbuminuria and hypertension with focus on type 1 and type 2 diabetes. J Intern Med 254:45-66

48. Hinokio Y, Suzuki S, Hirai M, Suzuki C, Suzuki M, Toyota T (2002) Urinary excretion of 8-oxo-7,8-dihydro-2'-deoxyguanosine as a predictor of the development of diabetic nephropathy. Diabetologia 45:877-882

49. van Hecke MV, Decker JM, Nijpels G et al (2005) Inflammation and endothelial dysfunction are associated with retinopathy: the Hoorn study. Diabetologia 48:1300-1306

50. Hjelmeland LM, Cristofolo VJ, Funk W, Rakoczy E, Katz ML (1999) Senescence of the retinal pigment epithelium. Mol Vis $5: 33$

51. Antcliff RJ, Marshall J (1999) The pathogenesis of edema in diabetic maculopathy. Semin Ophthalmol 14:223-232

52. Shelton DN, Chang E, Whittier PS, Choi D, Funk WD (1999) Microarray analysis of replicative senescence. Curr Biol 9:939-945

53. Honda S, Weigel A, Hjelmeland LM, Handa JT (2001) Induction of telomere shortening and replicative senescence by cryopreservation. Biochem Biophys Res Commun 282:493-498

54. Matsunaga H, Handa JT, Aotaki-Keen A, Sherwood SW, West MD, Hjelmeland B (1999) Beta-galactosidase histochemistry and telomere loss in senescent retinal pigment epithelial cells. Invest Ophthalmol Vis Sci 40:1-2

55. van Hecke MV, Decker JM, Nijpels G et al (2003) Retinopathy is associated with cardiovascular and all cause mortality in both diabetic and non diabetic subjects: the Hoorn study. Diabetes Care 26:2958-2961

56. Adaikalakoteswari A, Balasubramanyam M, Mohan V (2005) Telomere shortening occurs in Asian Indian type 2 diabetic patients. Diabet Med 22:1151-1156 